\title{
A review of the manufacturing process and infection rate of 3D-printed models and guides sterilized by hydrogen peroxide plasma and utilized intra-operatively
}

\author{
Graham Ka-Hon Shea ${ }^{1 *}$ (10, Kenneth Lap-Kei Wu² ${ }^{2}$ Iris Wai-Sum Li ${ }^{3}$, Man-Fai Leung ${ }^{1}$, Ada Lai-Ping Ko ${ }^{1}$, \\ Lane Tse ${ }^{1}$, Sherby Suet-Ying Pang ${ }^{4}$, Kenny Yat-Hong Kwann ${ }^{1}$, Tak-Man Wong ${ }^{1}$, Frankie Ka-Li Leung ${ }^{1}$ \\ and Christian Xinshuo Fang ${ }^{1,5}$
}

\begin{abstract}
3D printing in the context of medical application can allow for visualization of patient-specific anatomy to facilitate surgical planning and execution. Intra-operative usage of models and guides allows for real time feedback but ensuring sterility is essential to prevent infection. The additive manufacturing process restricts options for sterilisation owing to temperature sensitivity of thermoplastics utilised for fabrication. Here, we review one of the largest single cohorts of 3D models and guides constructed from Acrylonitrile butadiene styrene (ABS) and utilized intra-operatively, following terminal sterilization with hydrogen peroxide plasma. We describe our work flow from initial software rendering to printing, sterilization, and on-table application with the objective of demonstrating that our process is safe and can be implemented elsewhere. Overall, 7\% (8/114 patients) of patients developed a surgical site infection, which was not elevated in comparison to related studies utilizing traditional surgical methods. Prolonged operation time with an associated increase in surgical complexity was identified to be a risk factor for infection. Low temperature plasma-based sterilization depends upon sufficient permeation and contact with surfaces which are a particular challenge when our 3D-printouts contain diffusion-restricted luminal spaces as well as hollows. Application of printouts as guides for power tools may further expose these regions to sterile bodily tissues and result in generation of debris. With each printout being a bespoke medical device, it is important that the multidisciplinary team involved in production and application understand potential pitfalls to ensuring sterility as to minimize infection risk.
\end{abstract}

Keywords: 3D printing, Infection, Sterilization, ABS

\section{Background}

Advances in 3D printing has allowed for this technology to become increasingly prevalent across medical disciplines. In the context of facilitating surgical management, 3D-printed models can be an essential component of both pre-operative planning as well as intra-operative

*Correspondence: mailto:gkshea@hku.hk

'Department of Orthopaedics and Traumatology, The University of Hong

Kong, Hong Kong, Hong Kong

Full list of author information is available at the end of the article execution. Its main advantage lies in the capacity to display each patient's unique anatomy, thus allowing for more accurate surgical procedures, often with a corresponding decrease in operative time and reduction in morbidity. Fused deposition modelling (FDM) is the most common and least costly printing technique and utilizes thermoplastic filaments as printing materials by means of extrusion from a heated print nozzle onto a build platform. In the specialty of orthopaedics, 3D models can allow for visualization of bony anatomy and implant contouring, 
whilst guides can be created to direct osteotomies as well as screw entry sites [1].

As 3D models/guides intended for intra-operative application can come into direct contact with patient tissues, it is critical that sterility is ensured [2]. Polymers with melting temperature below $132^{\circ} \mathrm{C}$ are not compatible with steam sterilization as this would result in deformation $[2,3]$. Low temperature $\left(65^{\circ} \mathrm{C}\right)$ sterilization methods suitable for $3 \mathrm{D}$ printouts include ethylene oxide (EtO) gas and hydrogen peroxide plasma, with the latter technique preferred due to its absence of toxic by-products [4]. Hydrogen peroxide plasma is generated following excitation beyond the gaseous phase and achieves sterility by inducing free-radical formation [5].

A multidisciplinary team at our orthopaedic academic unit has been producing 3D models and guides in-house since 2015. This was initially confined to orthopaedic applications but we have since expanded to provide printouts for other surgical contexts. A total of 300 models have been rendered over the past four years, with 114 cases confirmed to have been utilized intra-operatively. For such cases, it is not reported in the literature whether terminal sterilization by means of hydrogen peroxide gas plasma is sufficient in preventing infection [6]. Here, we review details for cases requiring the application of 3D models and guides intra-operatively, identify cases subsequently developing surgical site infection, and identify risk factors for infection. Biocompatibility of finished models and guides was not considered for this study. In detailing the design, printing, and sterilization of 3D printouts as well as infection-related outcomes amongst this sizable cohort, we demonstrate that our production process is safe for continuation and may be adopted elsewhere.

\section{Methods}

\section{Patient identification and inclusion / exclusion criteria}

We documented all 3D-printed models originating from our centre that were designed, printed, and utilized intraoperatively between the period of January 2015 and June 2019. Three sources of records from our 3D printing centre, TSSU (Theatre Sterile Supplies Unit) and electronic clinical notes were cross-referenced to ensure for intraoperative use and to clarify the details of application. Exclusion was on the basis of models not being utilized intraoperatively / insufficient documentation with regards to their use, $<3$ months follow-up from the index operation and of models not utilizing ABS as the printing material (Fig. 1).

Patient demographics (age, sex), comorbidities (diabetes mellitus, active malignancy), pre-operative serum albumin and operation time with regards to eligible cases were retrieved from electronic patient records. Cases of postoperative infection were identified via reporting from the responsible operating surgeon and from electronic patient records.

\section{D printing, processing and sterilization}

An overview of the manufacturing process of 3D-printed models is shown in Fig. 2. Digital imaging and communications in medicine (DICOM) based patient data of CT

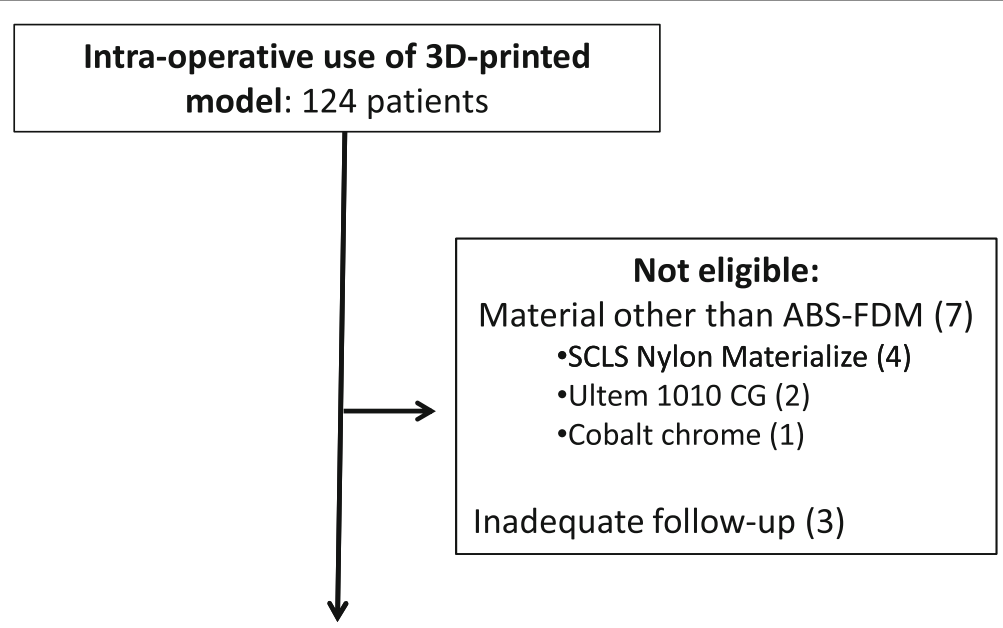

Eligible patients: 114 patients

Fig. 1 Patient eligibility and exclusion. More than 300 models were rendered by computer software from 2015 - 2019. The numbers of models proceeding to manufacturing, sterilization and intraoperative use amounted to 124. A further ten patients were excluded from analysis due to use of materials other than ABS (7 patients) as well as failure to reach 3-months of follow-up subsequent to surgery (3 patients), leaving a total of 114 patients eligible for analysis 


\section{Patient-specific 3D Model / Guide Processing Workflow}

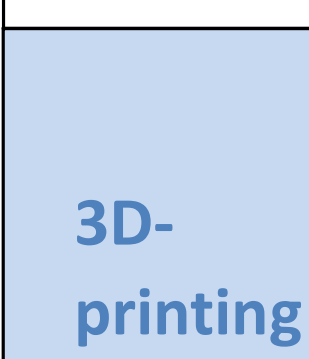

Retrieve patient data (DICOM CT) and ensure cuts are $\leq 0.625 \mathrm{~mm}$; convert to 3D model (STL file) using Mimics software

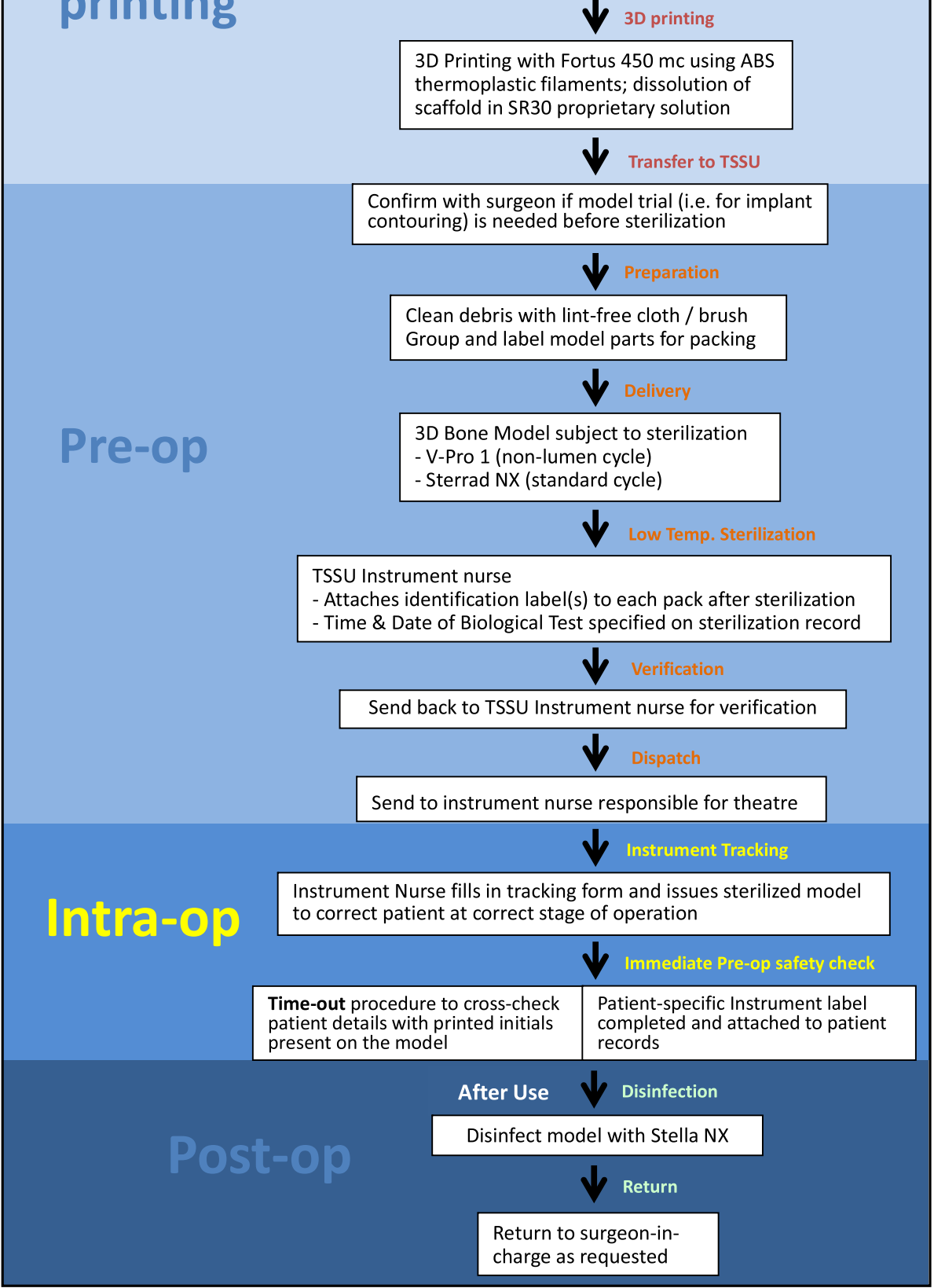

Fig. 2 Patient-specific 3D model / guide workflow. Overview of the model / guide production process from design to printing, sterilization, and intra-operative usage. DICOM = Digital Imaging and Communications in Medicine; STL = stereolithography (file format); TSSU = Theatre Sterile Supplies Unit 


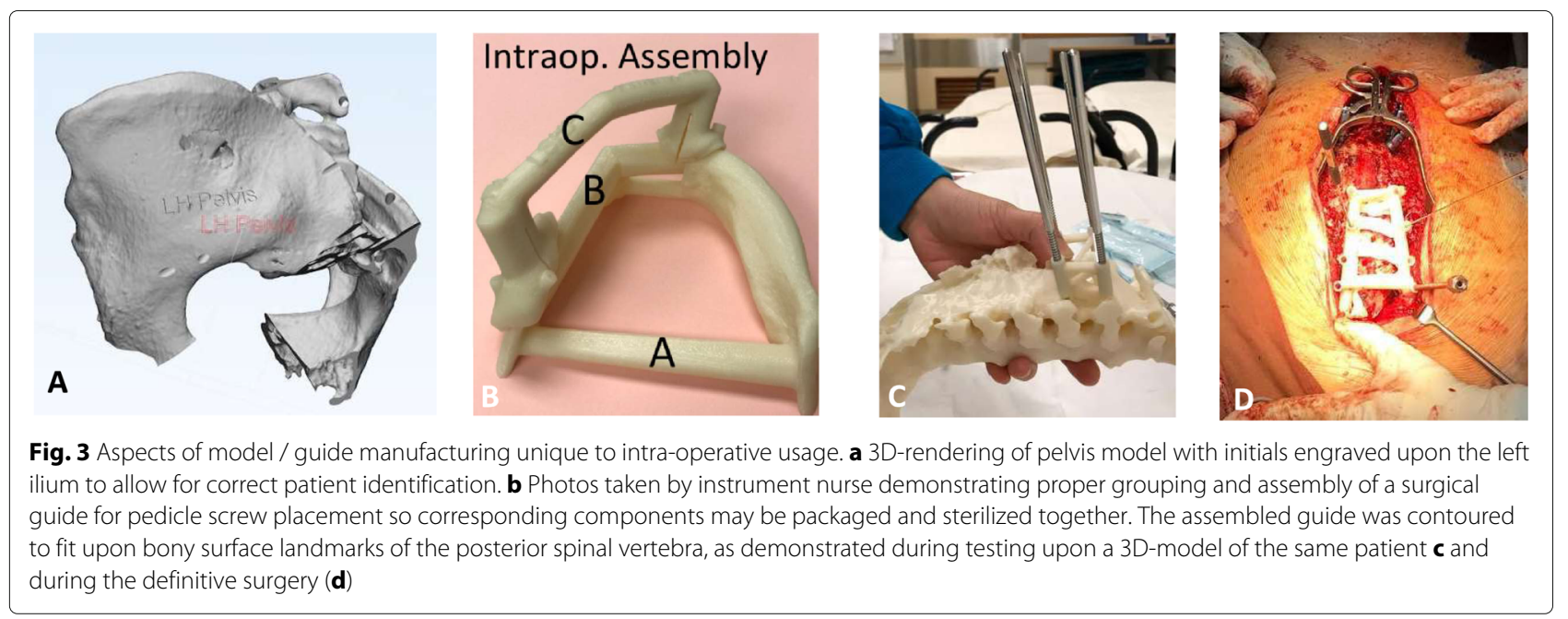

scans for the relevant bony region at $0.625 \mathrm{~mm}$ or finer slice thickness were recovered, segmented and processed by identification of relevant anatomical landmarks. These were subsequently converted into a digital 3D model in the stereolithography (STL) format using Mimics software version 20 or 21 (Materialise, Leuven Belgium). 3D models and guides were designed with Meshmixer (Autodesk, USA) and 3-Matic software (Materialise, Belgium). We added the corresponding patient's initials to non-essential regions of the printout to allow for on-table identification (Fig. 3a). The models and guides were manufactured with the FDM 3D printer Fortus 450mc (Stratasys, MI, USA) using the material ABS-M30i. Water soluble supporting material (SR30) utilized during the printing process was dissolved with WaterWorks ${ }^{\mathrm{TM}}$ dissolution mixture at $70^{\circ} \mathrm{C}$ for up to 24 hours.

Completed models / guides were cleansed with a lintfree cloth / brush to remove loose debris and rinsed

\section{B Bodily region for application}

\section{A Intended purpose of 3D printing}
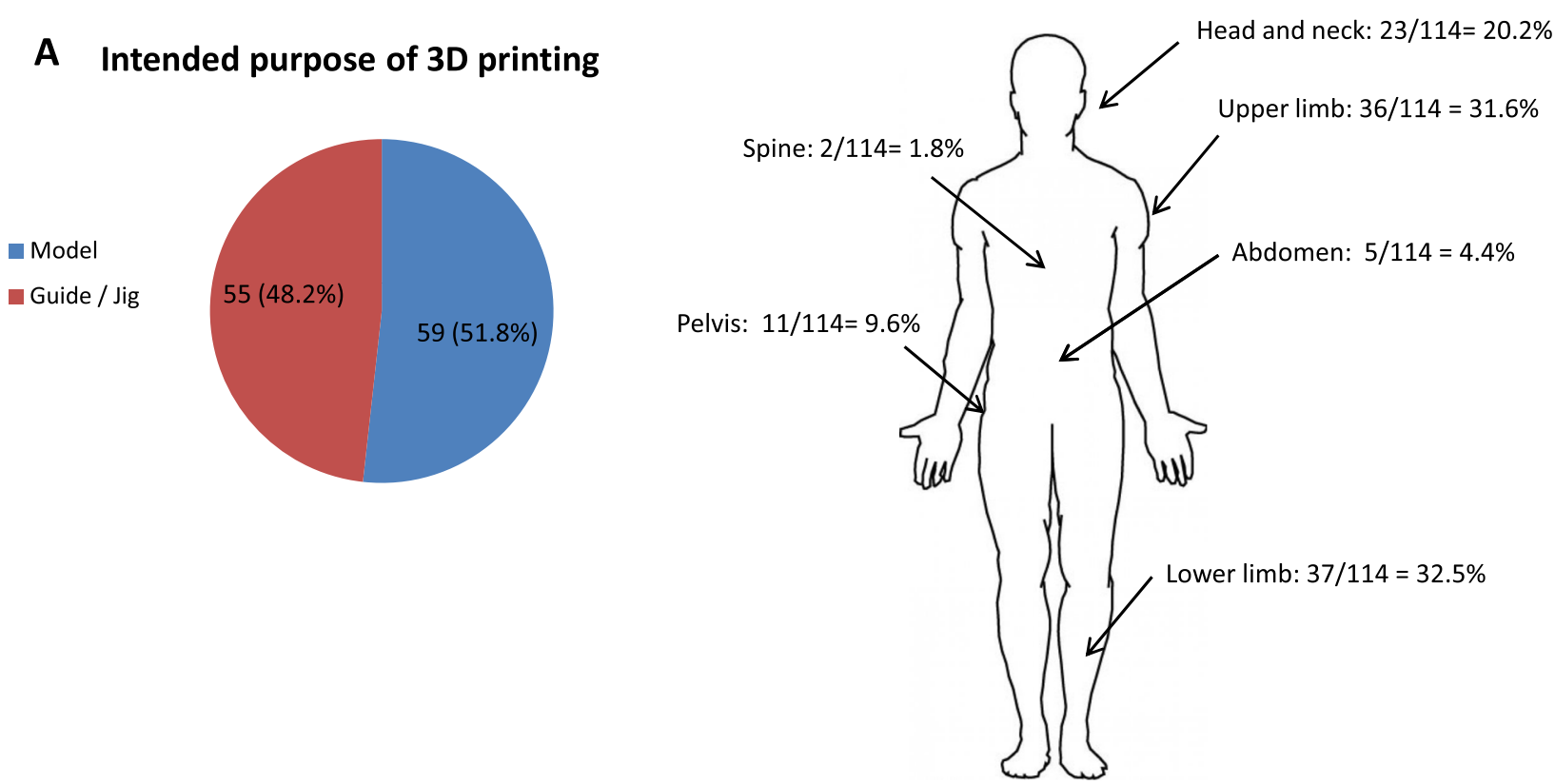

Fig. 4 Application of 3D printouts. a Intended purpose of 3D printout showing 59/114 (51.8\%) of printouts being utilized as anatomical models and $55 / 114$ (48.2\%) as guides / jigs intra-operatively. b The 124 cases utilized intraoperatively spanned different regions of the body as well as surgical specialties. The numbers relevant to each region and their percentages in relation to the whole patient cohort is shown 
with tap water. Printouts were air-dried before being packaged and subject to terminal sterilization. In the instance of models / guides with multiple components requiring assembly, the instrument nurse carefully photographed relevant groupings (Fig. 3b-d). Thereafter, grouped components were placed in labelled transfer boxes. Reprocessing records containing corresponding labels facilitated tracking of box contents. These steps enabled for relevant components to be packaged and sterilized together, and delivered to the surgeon intraoperatively in the correct combination and appropriate stage of surgery.

Printouts were sterilized with hydrogen peroxide plasma using either the V-Pro 1 Low Temperature Sterilization system (STERIS Corporation, Mentor, OH), or Sterrad ®System (Advanced Sterilisation Products, Irvine, CA). Low operating temperatures in both systems prevented material deformation during sterilization. For the V-Pro 1 Low Temperature system, we selected the pre-programmed non-lumen cycle. For the Sterrad @NX system we selected the pre-programmed standard cycle. Sterilized models / jigs were sent back to the instrument nurse for verification, stored until use, and then passed to the surgeon during the corresponding operation session. A surgical time-out procedure ensured that the printout was used for the correct patient, anatomical region and procedure, in accordance to initials upon the surface (Fig. 3a). Post-operatively, printouts were similarly subject to low temperature disinfection then returned to the surgeon in charge.

\section{Statistics}

Descriptive statistics were employed, with continuous variables expressed as mean \pm standard error of the mean (SEM). With regards to tests of statistical significance,
Fisher's exact test was used for comparison of categorical data, Student's T-test for comparison of continuous data between two groups, and ANOVA with post-hoc Tukey's test for multiple groups of continuous data. A $p$-value $<0.05$ (one-tailed) was taken to demonstrate statistically significant difference between groups.

\section{Results \\ Eligible cases and mode of application}

A total of 124 patients had 3D printouts that were utilized intra-operatively as part of their surgical management between the period of January 2015 and June 2019 (Fig. 1). Seven cases were excluded as printouts were not constructed from ABS, of which four cases utilized nylon, two case utilized polyetherimide (Ultem1010 CG), and one case utilized cobalt chrome. Three cases were excluded because of inadequate follow-up. A total of 114 models remained for subsequent analysis. Fifty nine out of 114 (51.8\%) were anatomical models utilized ontable for planning and / or implant contouring (Fig. 4A). The remaining 55/114 (48.2\%) were utilized as guides or jigs specific to patient anatomy to facilitate corrective osteotomies, screw insertion or pin placement. With regards to infection rate (Table 1A), 6/55 guides or jigs $(10.9 \%)$ and $2 / 59$ models (3.3\%) developed surgical site infections, which was not a statistically significant difference $(p=0.1141)$. All six cases of guides / jigs with infection were utilized to facilitate osteotomies. Both models with infection were utilized for implant contouring, one during fixation of a pilon fracture and the other for an orbital floor blowout fracture.

\section{Bodily region of involvement and operative details}

With reference to the bodily region that models / guides were utilized upon (Fig. 4B), 37/114 cases (32.5\%) involved

Table 1 (A) Table showing numbers of patients with infection according to mode of application as models or guides / jigs, which did not demonstrate statistically significant difference $(p=0.1141)$. (B) Infection rates according to bodily region of application, with numbers applied as models or guides / jigs in italics

\begin{tabular}{|c|c|c|c|c|}
\hline \multicolumn{5}{|l|}{ A } \\
\hline Mode of application & Infected cases & Non-infected cases & Total cases & Cases with infection (\%) \\
\hline Models & 2 & 57 & 59 & 3.4 \\
\hline Guide / jig & 6 & 49 & 55 & 10.9 \\
\hline \multicolumn{5}{|l|}{ B } \\
\hline Region & Infected cases (Models / Guides or jigs) & Non-infected cases (Models / Guides or jigs) & Total cases & Cases with infection (\%) \\
\hline Head \& Neck & $4(1 / 3)$ & $19(9 / 10)$ & 23 & $17.4^{*}$ \\
\hline Upper limb & 0 & $36(23 / 13)$ & 36 & 0 \\
\hline Lower limb & $4(1 / 3)$ & $33(23 / 10)$ & 37 & 10.8 \\
\hline Pelvis & 0 & $11(7 / 4)$ & 11 & 0 \\
\hline Abdomen & 0 & $5(0 / 5)$ & 5 & 0 \\
\hline Spine & 0 & $2(0 / 2)$ & 2 & 0 \\
\hline
\end{tabular}

${ }^{*} p=0.0195$ with regards to a significantly elevated infection rate of head and neck vs. upper limb cases 
the lower limb, 36/114 models (31.6\%) involved the upper limb, 23 cases $(20.2 \%)$ were in the head and neck region, 11 cases $(9.6 \%)$ in the pelvic region, five cases $(4.4 \%)$ as percutaneous soft tissue guides over the abdomen, and two cases $(1.8 \%)$ involved the spine. Surgical site infections (SSI) developed over the head and neck region $(4 / 23$; $17.4 \%)$ and lower limb $(4 / 37 ; 10.8 \%)$ but were absent in the other bodily regions. In performing statistical comparison between infection rates of operations in these respective bodily regions (Table 1B), there was a significant increase in head and neck cases with infection as compared to upper limb cases $(4 / 23$, vs. $0 / 36 ; p=0.0195)$. The most common surgical procedures requiring three-dimensional printouts together with their mode of application, infection rate and average operation times are listed in Table 2. The operative time for patients receiving mandible resection was significantly higher than those receiving reverse shoulder arthroplasty, pilon fracture ORIF, and tibial plateau ORIF $(p<0.01)$ although there was no statistical significant difference between infection rates $(p=$ 0.2792).

With regards to the details of the 37 cases involving the lower limb, 24/37 (64.9\%) were models used for intra-operative planning and/or plate contouring. These models were designed to facilitate surgical fixation of pilon fractures (nine patients), tibial plateau fractures (six patients), calcaneus fractures (four patients), ankle fractures (two patients), femoral shaft malunion (one patient), distal femur malunion (one patient) and metatarsal giant cell tumour (one patient). There were also 13 osteotomy guides $(35.1 \%)$ printed for use over the lower limb. Three guides were based around the knee, to address deformity subsequent to fracture malunion (two patients) and as a complication of tuberculosis (one patient). Guides facilitated osteotomies over the distal femur in two patients suffering from osteosarcoma. Four patients had tibial shaft osteotomy guides, correcting for fracture malunion (two patients), rickets (one patient), and pseudoarthrosis secondary to neurofibromatosis (one patient). There was one patient each receiving corrective osteotomies for malunions of the fibula, tibial plateau, midfoot and calcaneus.

For the 36 cases involving the upper limb, 13 (36.1\%) were models generated to facilitate intra-operative planning and/or plate contouring following fractures; of these seven were over the distal radius, two involved the mid-forearm, two involved the distal humerus, one involved the superior shoulder suspensory complex and one was over the proximal humerus. Another 23 (63.9\%) were guides, which in 14 patients facilitated glenoid guidepin and screw placement during reverse shoulder arthroplasty. The remaining nine printouts were utilized as osteotomy guides. Of these, five were to address malunion of the distal radius, three for malunion of the distal humerus, and one to guide resection of the clavicle in a patient with malignant fibrous histiocytoma.

Over the head and neck region the most common application of 3D-printing was to generate mandible osteotomy guides in the management of malignancies, accounting for $10 / 23$ cases $(43.5 \%)$. Seven contralateral mirror-imaged auricular models were generated and brought on-table to facilitate microtia surgical reconstruction, as well as two orbital wall models for implant contouring during fixation of blow-out fractures. Two models allowed for plate contouring following mandibular fractures. One guide was generated to facilitate burring of excess bone in a case of fibrous dysplasia affecting the maxilla. Another guide facilitated cranioplasty in the management of a skull osteoma.

A total of 11 printouts were located over the pelvic region. Specifically, seven models $(63.6 \%)$ were utilized for surgical planning, reduction and/or plate contouring for complex fractures affecting the pelvis and acetabulum. There were three cutting guides based around the pelvis, allowing for resection of acetabular metastasis, to facilitate conversion of a fused hip to arthroplasty, and for acetabular reshaping subsequent to malunion. There was also one screw insertion guide for sacroiliac fixation. Finally, five soft tissue guides were designed according to the patient's abdominal contour

Table 2 The five most common applications amongst our patient cohort are listed, together with their infection rate, need for re-operation, and average length of operation in minutes (mean \pm SEM)

\begin{tabular}{llllll}
\hline Operation performed & Mode of application & Number of cases & $\begin{array}{l}\text { Cases with } \\
\text { infection (\%) }\end{array}$ & Need for re-operation & $\begin{array}{l}\text { Average operation time } \\
\text { (minutes } \pm \text { SEM) }\end{array}$ \\
\hline Reverse shoulder arthroplasty & Guide & 14 & 0 & N/A & $169 \pm 17$ \\
Mandible resection & Guide & 10 & $2(20 \%)$ & 1 of 2 & $649 \pm 52^{*}$ \\
Pilon fracture ORIF & Model & 9 & $1(11.1 \%)$ & 0 of 1 & $193 \pm 17$ \\
Acetabular / pelvis fracture ORIF & Model & 7 & 0 & N/A & $400 \pm 159$ \\
Tibial plateau ORIF & Model & 6 & 0 & N/A & $122 \pm 23$ \\
\hline
\end{tabular}

* Patients receiving mandible resection had significantly longer operation time than those receiving reverse shoulder arthroplasty, pilon fracture ORIF, and tibial plateau ORIF $(p<0.01)$. ORIF = open reduction and internal fixation 
for cryoablation of renal cell carcinoma, and two cutting guides to the spine were fashioned for vertebral column resection in the context of scoliosis and Schuermann's kyphosis.

\section{Risk factors for infection}

A total of $8 / 114$ patients $(7.0 \%)$ had SSIs subsequent to intra-operative use of 3D printouts. A comparison of patient demographics and risk factors between cases with and without infection is shown in Table 3; 20 patients were excluded from analysis due to insufficient information upon patient records. The average age amongst patients with infection was lower than that of non-infected cases (45.3 vs 53.7 years old; $p=0.0348$ ). Amongst the eight cases of infection there were six males and two females and gender was not a significant risk factor $(p=0.197)$. The average operation time amongst cases with infection was $399 \pm 105$ minutes as compared to $241 \pm 24$ minutes, which was significantly longer $(p=0.0373)$. As a reflection of case complexity, $4 / 8$ cases with infection extended beyond 5 hours of operation time. With regards to co-existing comorbidities and nutritional status, neither diabetes mellitus $(p=0.200)$, active malignancy $(p=0.094)$ nor pre-operative albumin levels $(p=0.151)$ demonstrated statistically significant differences between infected and non-infected cases.

Details for individual cases with infection are shown in Table 4 . With regards to timing of presentation, $4 / 8$ cases presented within 6-weeks of surgery. Cases presenting within the first week post-op included a case of periorbital cellulitis subsequent to orbital floor fixation that resolved with antibiotics, and a case of pilon fracture fixation that presented with persistent post-op fever due to an infected hematoma that required debridement. Cases of late infection following more than 6-months postoperation presented as a superficial infection (cellulitis) for two patients and as a deep infection (osteomyelitis) for one patient. The case of late infection due to osteomyelitis was the most serious amongst those reported within our patient cohort. This patient had a history of diabetes mellitus and neurofibromatosis and received a corrective wedge osteotomy of the tibia and fibula followed by both internal and external fixation to confer additional stability. The patient presented at 7 -months post-operation and required multiple episodes of soft tissue and bone debridement for an infection involving the pin tracts tracking down to the tibia. Revision fixation and bone grafting of the resultant bony defect was required for reconstruction subsequent to debridement. Overall, surgical debridement and/or implant revision was required in $5 / 8(62.5 \%)$ patients with infection.

\section{Discussion}

We have designed and manufactured a large number of 3D printouts with their intra-operative application ranging from models for plate contouring to corrective osteotomy guides and jigs for screw placement. With printouts in direct contact with patient tissues, it is important that we are able to assess for the risk of infection [6]. The overall infection rate of $7 \%$ was comparable to figures in the literature using traditional surgical techniques. Our process for the production of models and guides for intraoperative application is safe, yet users need to be aware of potential caveats.

Additive manufacturing utilizing thermoplastic filaments may in itself ensure for sterility of the immediate printout as biomaterials are subject to temperatures in excess of $200^{\circ} \mathrm{C}$ [7]. Nevertheless a substantial amount of post-print processing is required, during which printouts are manipulated in non-sterile environments and therefore subject to contamination. Bacterial colonization resulting in biofilm formation upon ABS has been demonstrated [8], which enables for evasion from anti-microbials as well as host defences [9]. Roughness and topography are surface properties that influence biofilm formation, and of relevance to our printouts which reproduce irregular bony surfaces [10]. We have on occasion stored our printouts post-sterilization for weeks, or conversely proceeded to intra-operative utilization without a sufficient quarantine period post-sterilization, both of which may predispose to infection. Nevertheless formal regulation of design, manufacturing and sterilization processes will be difficult as each printout is a unique patient-specific

Table 3 Demographics and risk factors comparing infected and non-infected cases

\begin{tabular}{llll}
\hline Demographics / Risk factors & Infected cases & Non-infected cases & $P$-value \\
Age & $45.3 \pm 3.7$ & $53.7 \pm 2.1$ & $\mathbf{0 . 0 3 4 8}$ \\
Sex & Male -6 Female - 2 & Male -45 Female -41 & 0.197 \\
Operation time (minutes) & $399 \pm 105$ & $241 \pm 25$ & $\mathbf{0 . 0 3 7 3}$ \\
Diabetes mellitus & Yes $-3 \mathrm{No}-5$ & Yes -16 No -70 & 0.200 \\
Active malignancy & Yes $-3 \mathrm{No}-5$ & Yes -11 No -75 & 0.094 \\
Serum albumin $(\mathrm{g} / \mathrm{L})$ & $37 \pm 3.0$ & $41.3 \pm 0.5$ & 0.151 \\
\hline
\end{tabular}

Statistical comparison was carried out between relevant patient demographic details and risk factors for infection. Twenty cases were excluded from analysis due to incomplete medical records. With regards to analysed factors, younger age as well as longer operation times were identified as statistically significant risk factors for infection Sex, presence of diabetes mellitus, active malignancy and serum albumin (obtained from pre-operative liver function tests) were not correlated with infection risk 


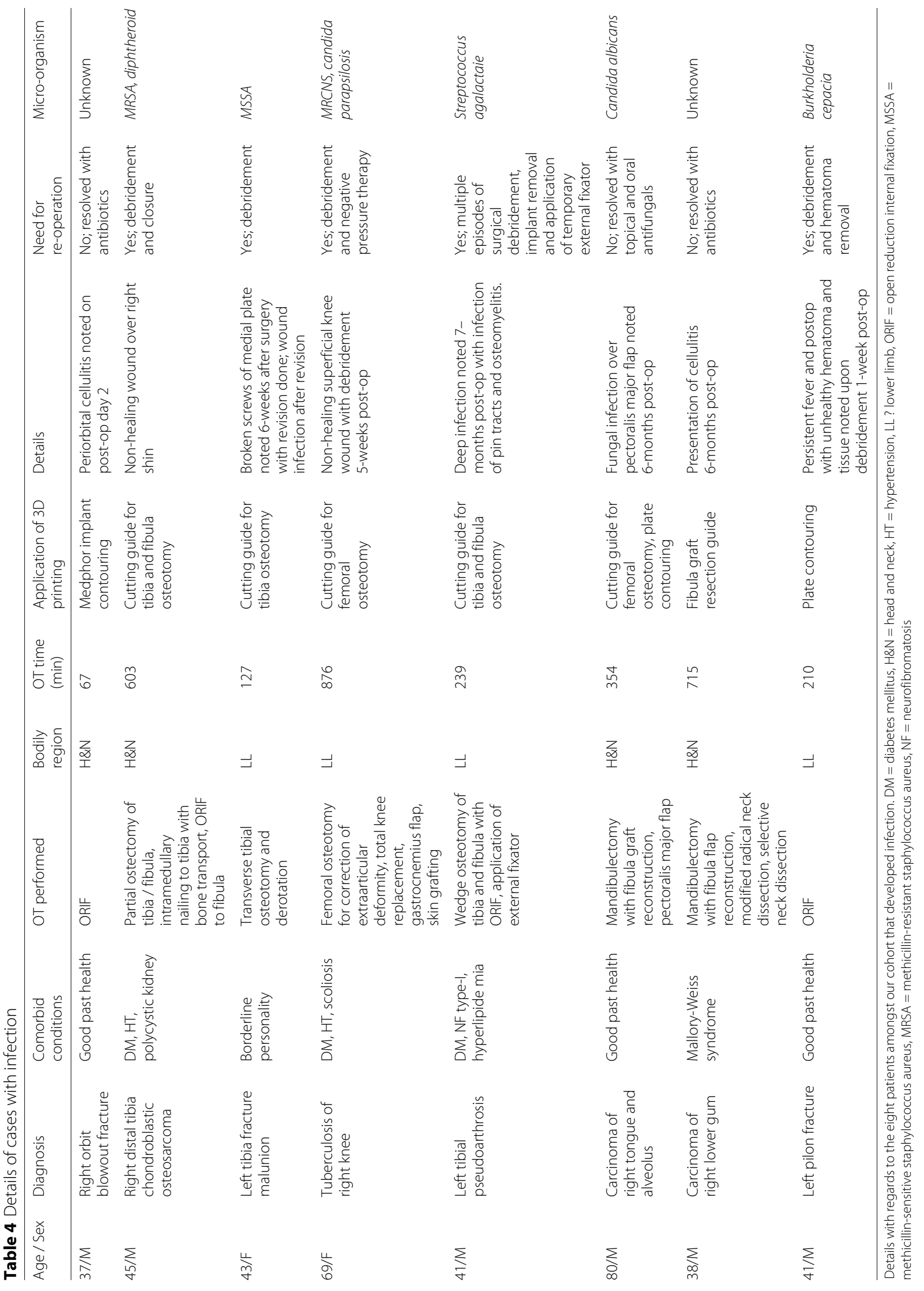


device [11]. Use of biological indicators can be a means of supporting adequate sterilization for each customized printout.

We predominantly used the V-Pro 1 low-temperature sterilization system for our heat-sensitive 3D printouts. This system utilizes hydrogen peroxide plasma to achieve sterility and is applicable across a range of heat- and moisture-sensitive materials. Sterilization is dependent upon plasma permeation and direct contact, with confined spaces involving long narrow lumens, for example during the sterilization of endoscopes, being potentially
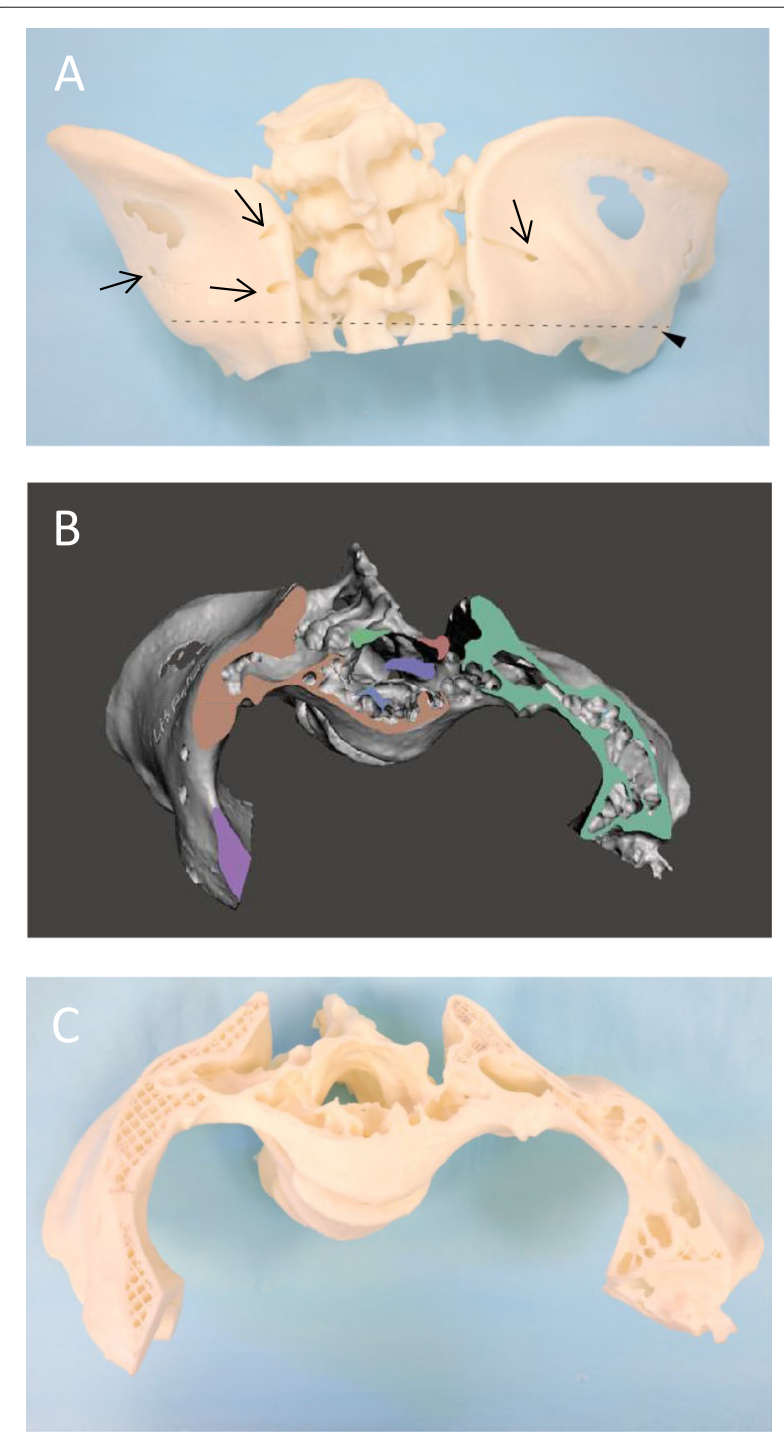

Fig. 5 Cross-section of pelvis 3D model demonstrating irregular luminal spaces. a Arrows indicate surface openings upon the posterior ilium on a 3D model of the pelvis. The dotted line and arrowhead demonstrates the level of transverse sectioning subsequently performed. Cross-sectional appearance following software rendering $\mathbf{b}$ and physical sectioning $\mathbf{c}$ of the same pelvis model demonstrating irregular trabecular spaces contained within problematic. Under controlled testing however, plasma sterilization eradicated bacteria spores seeded to a depth of $500 \mathrm{~mm}$ within $0.7 \mathrm{~mm}$ diameter tubing [12]. It is uncertain how hydrogen peroxide plasma will penetrate into narrow lumen (Fig. 5a) leading to irregular trabecular spaces (Fig. 5b and c) that correspond to cancellous bony structure. Many of these spaces are enclosed and do not directly communicative with the external environment to allow for gas permeation and risks contamination subsequent to intra-operative exposure. Biological indicators may be embedded within the complex internal structure as a form of stress testing. Alternately in being non-essential for function, internal structural motifs could be simplified in design prior to printing. Nevertheless, plasma based methods would be advantageous for the permeation of internal structures in comparison to surface sterilization of heat-sensitive materials by ultraviolet light [13]. Seperately, removal of supporting material by heating under alkali conditions may be incomplete [14] thus shielding ABS from sterilization.

Tissue biocompatibility is an additional factor that merits discussion. Due to contact between exposed tissues and printouts as well as debris generated by intra-operative damage (Fig. 6) it is important to be able to ensure for the safety of such medical devices by biocompatibility testing. Developed by the International Organization of Standardization, ISO 10993 describes a series of standards for biocompatibility testing including cytotoxicity assays, assays for irritation and skin sensitisation, as well as guidelines for sample extraction. ABS-M30i complies with ISO 10993 standards yet this is with regards to its raw form prior to printing and post-print processing. Inspection of damage to guides / jigs, removal of visible fragments over the surgical site, and copious irrigation of exposed tissues would provide a simple means to reducing cytotoxicity and debris retention. We did see a higher proportion of infections in the application of printouts as guides / jigs as compared to models. Nevertheless this increase in infection rate could also be the result of lengthier and more complex operations, as we have demonstrated operative time to be a risk factor. The younger age of patients with infection as compared to those without was an unexpected finding, but likely due to the management decision for complex curative surgery and reconstruction amongst those suffering from malignancy when age and co-morbidities were not contraindications for a major operation.

With regards to the benefits of 3D modelling on surgical execution, a meta-analysis compiling studies for pilon fracture management reported that operation time, blood loss, post-operative pain scores, achievement of anatomical fracture reduction, and post-operative function were all improved in comparison to conventional planning [6]. Rates of infection following 3D modelling 


\section{A}

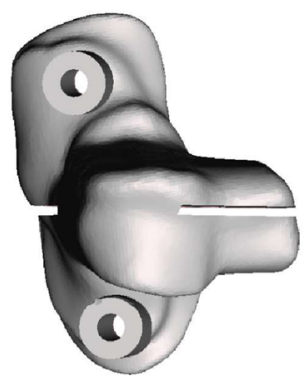

D

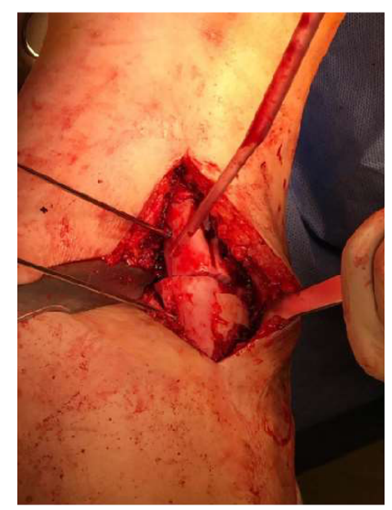

B

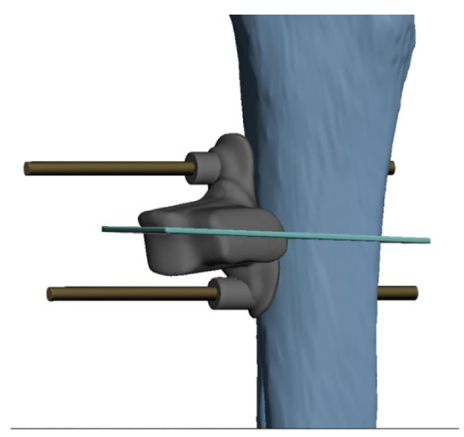

E

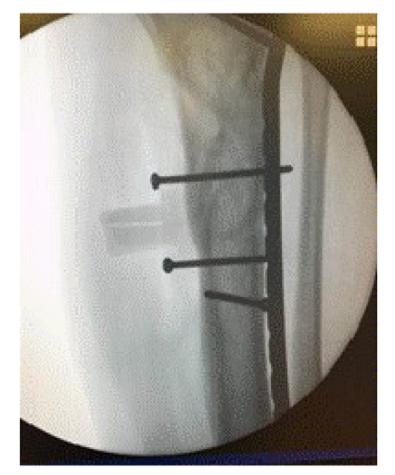

C

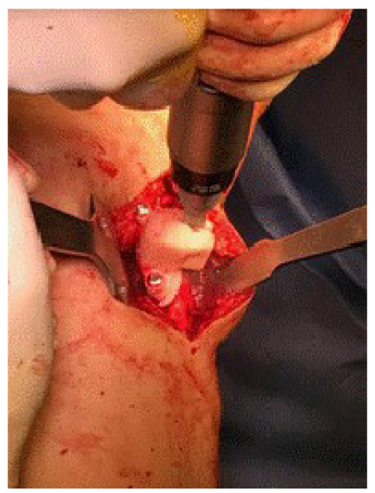

$\mathbf{F}$

Fig. 6 Intraoperative use of osteotomy guide. a Software rendered image of guide intended for corrective osteotomy and shown upon the tibial shaft (b) with the osteotomy site marked in teal. c Intra-operative photo with the guide secured and oscillating saw engaged in preparation for osteotomy and (d) upon completion. e Intra-operative x-ray demonstrating reduction and fixation of the tibial shaft following corrective osteotomy. f Similar guide retrieved post-op demonstrating damage to ABS over the osteotomy slit with the potential to release debris

amongst this cohort were not increased, and have been reported elsewhere to be as high as $16.1 \%$ owing to the degree of associated soft tissue trauma, with an average presentation time of 88-days post-op [15]. Amongst out patients with pilon fractures, $1 / 9(11.1 \%)$ developed infection. Another study utilized 3D-printed ABS models to facilitate fixation of complex tibial plateau fractures in 69 patients [16]. A total of $6 / 69$ (8.7\%) suffered from SSIs, whilst within our cohort none of the six cases did. Conventional reverse shoulder arthroplasty has a reported infection rate of approximately 5\% [17], and amongst our cohort none of the 14 patients receiving this procedure in conjunction with 3D-printed guides for glenoid guidepin / screw placement had a surgical site infection within 3-months. Mandibular resection and reconstruction in the treatment of malignant tumours is a formidable challenge with complication rates approaching 70\% [18] and in our cohort head and neck cases $(4 / 23 ; 17.4 \%)$ represented the region with the highest infection rate. We did not see evidence in the cases with infection indicating the persistence of sterilization-resistant microorganisms such as bacterial spores [19] following hydrogen peroxide plasma treatment. It is worth noting that prior studies detailing infection-related outcomes of 3D printouts have not explicitly utilized them intra-operatively, and this is one of the first studies to have done as such. Our overall impression was that our process of sterilization and on-table usage is safe, and that surgical complexity and tissue manipulation as reflected by increased operating time were the main culprits for infection.

Limitations to this study included its retrospective design without a designated control group, and insufficient power for detailed subgroup analysis. Designating 3-months of follow-up as an inclusion criterion may have failed to account for infections presenting at a later phase. It would be of interest to report on an increased cohort size over a longer period of follow-up at our center as the process continues to mature, in order to account for these present deficiencies.

\section{Conclusion}

We have described one of the largest patient cohorts to receive intra-operative application of 3D models / guides. 
Our present workflow for the manufacturing, sterilization and intra-operative application of 3D models is safe for continuation as well as implementation elsewhere. With each printout being a bespoke medical device, it is essential for users to be aware of the unique structural properties of models / guides as well as constraints in the production process with regards to infection and biocompatibility. We forsee that the scope of application during surgery can only expand once the production process is established to be reproducible, consistent and safe.

\section{Abbreviations}

ABS: Acrylonitrile butadiene styrene; DM: Diabetes mellitus; FDM: Fused deposition modelling; EtO: Ethylene oxide; HT: Hypertension; MSSA: Methicillin-sensitive staphylococcus aureus; MRSA: Methicillin-resistant staphylococcus aureus; MRCNS: Methicillin-resistant coagulase-negative staphylococci; NF: Neurofibromatosis; ORIF: Open reduction internal fixation; SSI: Surgical site infection; TSSU: Theatre Sterile Supplies Unit

\section{Acknowledgements}

We would like to acknowledge the Tam Shiu Charitable Trust for their generous support.

\section{Authors' contributions}

GS - collected and analyzed the data, wrote the manuscript; KW, MF collected the data; IS, AK, LT - provided critical revision of the article; SP, KK, TM, FK - performed the surgery and provided critical revision of the article; CF contributed to conception and design of the study, funding, and critical revision of the article. All authors read and approved the final manuscript.

\section{Funding}

$3 \mathrm{D}$ rendering and printing was supported by funding from the Tam Shiu Charitable Trust awarded to Dr Christian Fang.

\section{Ethics approval and consent to participate}

Informed consent was obtained from all individual participants included in the study. We received ethical approval for intra-operative use of 3D-printed guides and models via the Institutional Review Board of the University of Hong Kong / Hospital Authority Hong Kong West Cluster.

All procedures performed in studies involving human participants were in accordance with the ethical standards of the institutional and/or national research committee and with the 1964 Helsinki declaration and its later amendments or comparable ethical standards.

\section{Consent for publication}

Not applicable.

\section{Competing interests}

None of the authors have conflicts of interest to declare.

\section{Author details \\ ${ }^{1}$ Department of Orthopaedics and Traumatology, The University of Hong Kong, Hong Kong, Hong Kong. ${ }^{2}$ School of Biomedical Sciences, The University of Hong Kong, Hong Kong, Hong Kong. ${ }^{3}$ School of Public Health, The University of Hong Kong, Hong Kong, Hong Kong. ${ }^{4}$ Department of Surgery, The University of Hong Kong, Hong Kong, Hong Kong. ${ }^{5}$ Tam Shiu Foundation Anatomical Modelling Laboratory, Hong Kong, Hong Kong.}

Received: 18 November 2019 Accepted: 10 March 2020

Published online: 30 March 2020

\section{References}

1. Galvez M, Asahi T, Baar A, Carcuro G, Cuchacovich N, Fuentes JA, Mardones R, Montoya CE, Negrin R, Otayza F, et al. Use of three-dimensional printing in orthopaedic surgical planning. J Am Acad Orthop Surg Glob Res Rev. 2018;2(5):e071. https://doi.org/10.5435/ jaaosglobal-d-17-00071.
2. Rutala WA, Weber DJ. Disinfection and sterilization: an overview. Am J Infect Control. 2013;41(5):S2-5.

3. Ngo TD, Kashani A, Imbalzano G, Nguyen KTQ, Hui D. Additive manufacturing (3D printing): A review of materials, methods, applications and challenges. Compos B Eng. 2018;143:172-196.

4. Boiano JM, Steege AL. Ethylene oxide and hydrogen peroxide gas plasma sterilization: Precautionary practices in US hospitals. Zentralsterilisation (Wiesbaden). 2015;23(4):262.

5. McEvoy B, Rowan NJ. Terminal sterilization of medical devices using vaporized hydrogen peroxide: a review of current methods and emerging opportunities. J Appl Microbiol. 2019;127(5):1403-20.

6. Bai J, Wang Y, Zhang P, Liu M, Wang P, Wang J, Liang Y. Efficacy and safety of 3D print-assisted surgery for the treatment of pilon fractures: a meta-analysis of randomized controlled trials. J Orthop Surg Res. 2018;13(1):283

7. Neches RY, Flynn KJ, Zaman L, Tung E, Pudlo N. On the intrinsic sterility of 3D printing. PeerJ. 2016:4:e2661.

8. Desrousseaux C, Cueff R, Aumeran C, Garrait G, Mailhot-Jensen B, Traoré $\mathrm{O}$, Sautou V. Fabrication of acrylonitrile-butadiene-styrene nanostructures with anodic alumina oxide templates, characterization and biofilm development test for Staphylococcus epidermidis. PloS one. 2015;10(8):e0135632.

9. Roberts CG. The role of biofilms in reprocessing medical devices. Am J Infect Control. 2013;41(5):S77-80.

10. Chung KK, Schumacher JF, Sampson EM, Burne RA, Antonelli PJ, Brennan AB. Impact of engineered surface microtopography on biofilm formation of Staphylococcus aureus. Biointerphases. 2007;2(2):89-94.

11. Morrison RJ, Kashlan KN, Flanangan CL, Wright JK, Green GE, Hollister SJ, Weatherwax KJ. Regulatory considerations in the design and manufacturing of implantable 3D-printed medical devices. Clin Transl Sci. 2015;8(5):594-600.

12. Diab-Elschahawi M, Blacky A, Bachhofner N, Koller W. Lumen claims of the STERRAD 100NX sterilizer: testing performance limits when processing equipment containing long, narrow lumens. Am J Infect Control. 2011;39(9):770-4.

13. Dunne $C P$, Jeremiah O'Sullivan KJ, O'Sullivan L, Linnane B, O'Connell $\mathrm{NH}$. Foreseeing the microbiology of bespoke 3D printed medical devices. 2018;99(2):237-238.

14. Scalfani VF, Williams AJ, Tkachenko V, Karapetyan K, Pshenichnov A Hanson RM, Liddie JM, Bara JE. Programmatic conversion of crystal structures into 3D printable files using Jmol. J Cheminformatics. 2016;8(1): 66

15. Molina CS, Stinner DJ, Fras AR, Evans JM. Risk factors of deep infection in operatively treated pilon fractures (AO/OTA: 43). J Orthop. 2015;12:S7-13.

16. Wu W-y, Xu W-g, Wan C-y, Fang M. Preoperative Plan with 3D Printing in Internal and External Fixation for Complex Tibial Plateau Fractures. Orthop Surg. 2019;11(4):560-8.

17. Trappey GJ, O'connor DP, Edwards TB. What are the instability and infection rates after reverse shoulder arthroplasty?. Clin Orthop Relat Res. 2011;469(9):2505-11.

18. Wei F-c, Celik N, Yang W-g, Chen I-h, Chang Y-m, Chen H-c, et al. Complications after reconstruction by plate and soft-tissue free flap in composite mandibular defects and secondary salvage reconstruction with osteocutaneous flap. Plast Reconstr Surg. 2003;112(1):37-42.

19. Shintani H, Sakudo A, Burke P, McDonnell G. Gas plasma sterilization of microorganisms and mechanisms of action. Exp Ther Med. 2010;1(5): $731-8$

\section{Publisher's Note}

Springer Nature remains neutral with regard to jurisdictional claims in published maps and institutional affiliations. 